${ }^{3}$ Ruderman, M., Vature, 218, 1128 (1968).

${ }^{4}$ Fritz, G., et al., Science, 164, 709 (1969).

${ }^{5}$ Reichley, P., and Downs, G., Nature, 222, 299 (1969); Radhakrishnan, V., and Yanchester, P., ibid.,222, $298(1969)$.

${ }^{6}$ Frank, F. C., Nature, 220, 350 (1968).

\section{Pulsar Slowdown Rates for CP 0328 and HP 1506}

Trming observations of pulsars $C P \quad 0328$ and $H P 1506$ have been made with the four acre, $81.5 \mathrm{MHz}$, array of the Mullard Radio Astronomy Observatory since June 1968.

The equipment and technique used to refer the observed pulse arrival times to their arrival times at the solar system barycentre have been described before ${ }^{1}$. The only change has been that 1969 observations were taken with a crystal-controlled receiver with the accurate and stable $30 \mathrm{kHz}$ i.f. bandpass needed to overcome the errors resulting from frequeney structure and the high pulse dispersion of these pulsars at $81.5 \mathrm{MHz}$.

I'uble 1. PARAMETERS OF THE STOWDOWN

\begin{tabular}{|c|c|c|}
\hline Pulsar & $C P 0328$ & HP 1506 \\
\hline $\begin{array}{l}a(1950 \cdot 0) \\
\delta(1950 \cdot 0)\end{array}$ & $\begin{array}{l}03 \mathrm{~h} 29 \mathrm{~m} 11 \cdot 0 \mathrm{~s} \\
54^{\circ} 24^{\prime} 37 \cdot 2^{\prime \prime}\end{array}$ & $\begin{array}{l}15 \mathrm{~h} 08 \mathrm{~m} 3.27 \mathrm{~s} \\
55^{\circ} 42^{\prime} 50^{\prime \prime}\end{array}$ \\
\hline $\begin{array}{l}\text { Period April 1, } 1969 \\
\text { (A1 seconds) }\end{array}$ & $\begin{array}{c}0.714518603 \\
\pm 15 \mathrm{~ns}\end{array}$ & $\begin{array}{l}0.739677766 \\
\pm 10 \mathrm{~ns}\end{array}$ \\
\hline $\begin{array}{c}\text { Period change ns } \mathrm{y}^{-2} \\
\qquad P / P \\
\dot{P}\end{array}$ & $\begin{array}{c}60 \pm 5 \\
(1 \cdot 4 \pm 0 \cdot 1) \times 10^{-16} \\
(1.9 \pm 0.2) \times 10^{-15}\end{array}$ & $\begin{array}{c}167 \pm 14 \\
(3 \cdot 9 \pm 0 \cdot 3) \times 10^{-15} \\
(5 \cdot 3 \pm 0 \cdot 4) \times 10^{-15}\end{array}$ \\
\hline
\end{tabular}

Table 1 lists the assumed positions (A. G. Lyne, Herstmonceux Conf. on Astrometry, April 1969) used to derive the plots of pulsar phase which show $C P 0328$

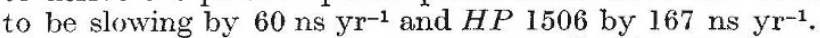
The parameters of this slowing are also listed in Table 1.

If pulsars derive their pulsation energy from stellar rotation, a simple relation might be expected between the rate of increase of the period and the period itself ${ }^{2,3}$. The present results, in agreement with previous observations (refs. 1, 4 and A. G. Lyne at the Herstmonceux Conf.), show that no such simple relation exists for pulsars having periods longer than about $0.25 \mathrm{~s}$. It follows that the simple rotational models have parameters which vary considerably from source to source. Such parameters might, for example, be the strength of the stellar magnetic dipole moment, the inclination between the rotation axis and the magnetic axis, conditions in the plasma surrounding the star or the rates of possible variation of these parameters with time.

A relationship between rate of ehange of period and period itself might then be found only if slowdown rate could be combined with the other observed quantities of the pulsar in a detailed source model.

I thank $H$. Hesse for help and CSIRO for a research scholarship.

Note added in proof. Since submitting, I have been informed that independently found values at Jodrell Bank agree with these results.

T. W. COLE

Mullard Radio Astronomy Observatory,

University of Cambridge.

Received June 23, 1969.

1 Cole, T. W., Nature, 221, 29 (1969)

2 Pacini, F, Nature, 219, 145 (1968).

${ }^{3}$ Gunn, J. E., and Ostriker, J. P., Nature, 221, 454 (1969).

- Davies, J. G., Hunt, G. C., and Smith, F. G., Nature, 221, 27 (1969)

\section{Interstellar Hydrogen Atoms on Graphite Grains}

DuLEX ${ }^{1}$ has recently suggested that the cause of the deficiency of the stellar ultraviolet flux below the Lyman- $\alpha$ line may be due to hydrogen atoms near interstellar grains absorbing at wavelengths shifted from $1216 \AA$. Duley has calculated the shift in the $1 s$ and $2 p$ levels of an $\mathrm{H}$ atom near a pure dielectric grain by placing the atom at the centre of a spherical cavity of radius $R_{0}$ in a medium specified only by a dielectric constant $K$. For $K=1 \cdot 1 \rightarrow 1 \cdot 4$ and $R_{0}=2 \rightarrow 5 a_{0}$, the resultant shift in the Lyman- $\alpha$ line is positive, and on the order of a few tenths of an electron volt, so that absorption would occur at wavelengths shorter than $1216 \AA$.

The material of the interstellar grains is frequently assumed to be graphite ${ }^{2}$ which in many respects behaves more as a metal than a dielectric. Notably, it is a good conductor in the plane of the crystal. We have derived expressions for energy shifts in one and two-effective electron atoms near metals; this work will be presented elsewhere. Using these results we have calculated the energy shifts in the $1 s$ and $2 p$ states of an $\mathrm{H}$ atom at various distances from a plane graphite surface, treated as a metal. The atom-metal interaction, $H^{\prime}$, is calculated by image-potential theory, and the energy shift in the $n l$ level is calculated from first order perturbation theory, thus

$$
\Delta E(n l)=\left\langle n l\left|H^{\prime}\right| n l\right\rangle \mid\langle n l \mid n l\rangle
$$

where the region of integration is a truncated infinite region, outside the metal. Expressions given by equation (1) are cumbersome and will not be presented. In Fig. I we show graphs of $\Delta E(1 s)$ and $\Delta E(2 p)$ as functions of the atom-metal distance.

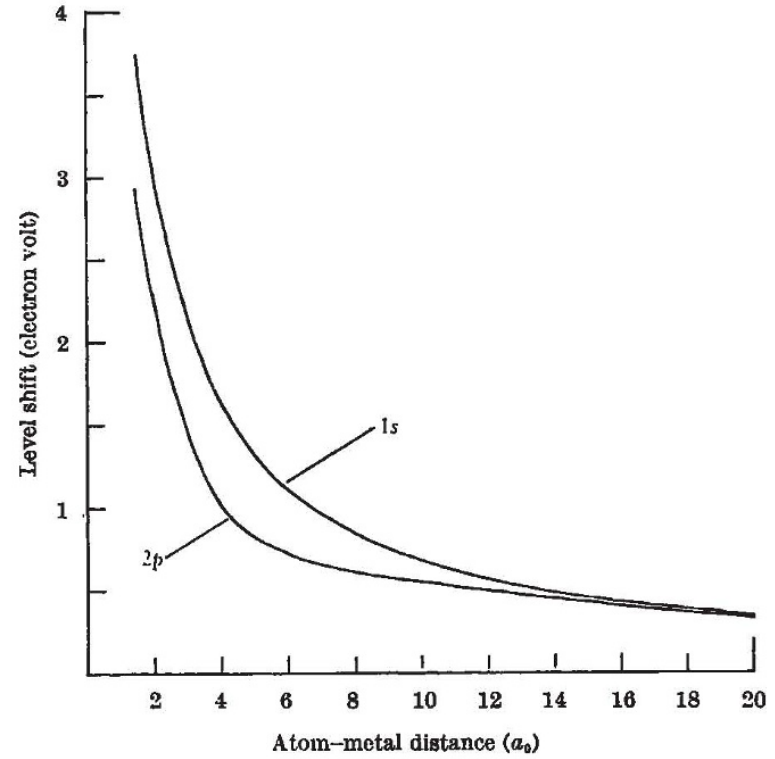

Fig. 1. The shift in electron volts of the $1 s$ and $2 p$ levels of an $\mathrm{H}$ atom perturbed by the proximity of a metal surface, as a function of atommetal distance in $a_{0}$.

Both shifts are positive, and $\Delta E(1 s)>\Delta E(2 p)$ for all atom-metal distances considered. An examination of the form of $H^{\prime}$ in relation to the shape of the wave functions makes clear why the groundstate shift is larger.

It seems that if an $\mathrm{H}$ atom is associated with a graphite grain which retains appreciable crystal structure, then the resonance line is shifted to wavelengths longer than $1216 \AA$, by about $50 \AA$ for a typical atom-metal distance of $6 a_{0}$. 